\title{
Book Reviews / Comptes rendus
}

\section{Pascale Mounier}

\section{Le Roman humaniste : un genre novateur français (1532-1564)}

Paris : Honoré Champion, 2007, 506 p.

Définir le roman est une entreprise ardue ; le recours à la prose, à la notion de longueur et au concept de personnages constituent en effet des critères approximatifs et subjectifs. Dès lors, deux possibilités s'offrent au critique : soit il dresse empiriquement une typologie, qu'il tentera d'ordonner et d'enrichir au gré de ses lectures, soit il adopte, après les travaux des formalistes russes notamment, l'hypothèse selon laquelle il existe des invariants, qu'il s'applique à identifier aussi bien au niveau de la microstructure que de la composition générale des œuvres étudiées. C’est le parti pris par P. Mounier, dont le dessein est de s'interroger sur l'émergence et les manifestations du genre romanesque au XVI siècle. C'est dire l'importance de ce travail, qui vient combler une lacune, sensible dès que l'on est confronté aux récits de la Renaissance. Un point d'ordre méthodologique mérite d'être précisé : l'auteur, qui a couvert un vaste territoire littéraire et critique, a retenu essentiellement les œuvres de François Rabelais, d'Hélisenne de Crenne, de Guillaume des Autels et de Barthélemy Aneau pour conduire sa démonstration. Le fait peut surprendre dans la mesure où ces textes, par ailleurs hétérogènes, paraissent alors isolés du reste de la production romanesque de cette période. En réalité, comme l'explique P. Mounier (432), c'est parce qu'elles portent à leur plus haut point de complexité et de richesse les différents problèmes liés à la question abordée ici que la prééminence a été accordée à ces œuvres. Trois grands moments ponctuent cette enquête, qui s'ouvre sur une analyse lexicologique des emplois du mot « roman $\gg(37-208)$, puis s'attache à mettre au jour l'originalité du genre « nouvelle manière » (209-318), et appréhende enfin les « enjeux cognitifs » liés à la présence de discours concurrents au sein de ce que l'auteur se propose de nommer le « roman humaniste » (319-444).

L'étendue sémantique des occurrences du mot « roman $\gg$ suscite un examen minutieux des « flottements linguistiques » qui affectent son emploi, qu'il s'agisse d'expressions ou de termes dont l'acception est plus étendue que « roman $»$, ou de désignations synonymiques. Sont déterminées ensuite les différents significations qui lui sont données au XVI ${ }^{\mathrm{e}}$ siècle dans le contexte littéraire, le roman étant alors considéré comme un récit inventé, marqué du sceau de la thématique chevaleresque et amoureuse. L'auteur rappelle aussi fort opportunément que Joachim Du Bellay, Jacques Pelletier et Pierre de Ronsard associent le roman à une épopée moderne fondée peu ou prou sur une trame chevaleresque. Lexicologique et diachronique, 
l'approche est également idéologique, dans la mesure où le terme « roman » est associé chez certains érudits à des œuvres jugées frivoles, tandis que d'autres louent la richesse de la matière et du langage hérités du substrat breton. Poursuivant son enquête, P. Mounier rend compte des lacunes concernant les canons littéraires du genre romanesque au $\mathrm{XVI}^{\mathrm{e}}$ siècle et, après avoir évoqué les polémiques auxquelles sa définition a donné lieu en Italie, expose en quoi consiste le travail de modernisation et de francisation de la matière romanesque. Il apparait alors que, tout en se situant dans le sillage des méthodes médiévales, cette activité de « translation » et d'adaptation infléchit significativement les sujets et les formes des modèles anciens, les «translateurs » n'hésitant pas, par le jeu de l'appropriation d'une langue et l'exhibition d'une certaine désinvolture à l'égard du texte-source, à construire un univers plus subjectif, où la notion de plaisir à faire naître chez le lecteur peut permettre d'appréhender d'une manière pertinente la théorie romanesque de la Renaissance.

Une fois mis en évidence le processus de francisation de la matière romanesque, l'étude se concentre sur l'originalité foncière du roman humaniste, « qui fait du langage, dans sa pluralité, l'objet de la narration $\gg$ (209). Quittant le domaine des théoriciens littéraires pour celui des auteurs, P. Mounier relève encore bien des approximations dans les termes convoqués pour désigner le roman, avec cependant parfois des substituts précis, tels « histoire veridicque » chez Rabelais ou « histoire fabuleuse » chez Aneau. De plus, deux notions fondamentales sont mises en exergue, celle de varietas et, sous l'impulsion d'Érasme, celle de copia. L’analyse permet ainsi d'identifier les procédés d'ornementation et d'amplification convoqués par les auteurs, tant sur le plan lexical, avec les latinismes, les polynômes synonymiques et les hyperboles, que syntaxique, avec les répétitions de construction ou les parallélismes. Soucieux d'embrasser les différents éléments constituant l'œuvre romanesque, l'auteur tente ensuite de mesurer l'impact de cette intrusion de styles hétérogènes sur la conduite du récit : copia et varietas ne risquent-elles pas à terme de faire voler en éclat la trame narrative, de tuer l'histoire ? Approfondissant son analyse, P. Mounier parvient à des conclusions fermes et claires, qui peuvent nous guider : le roman humaniste est fondé sur la juxtaposition de langages multiples et divers ; il révèle la présence d'un travail véritable sur la composition ; il maintient la représentation du réel grâce à des emprunts aux modèles antérieurs. Mais le point important réside dans la notion de mimèsis, qui ne doit pas être envisagée comme une imitation de la vie quotidienne, mais comprise « en termes de construction d'une référence feinte et subjective $\gg(317)$. 
La troisième partie de l'ouvrage s'intéresse aux enjeux cognitifs de la facture romanesque et à ses conséquences sur le plan idéologique et herméneutique. Désormais, l'instance scripturale est brouillée, et l'intervention massive et forte des personnages leur confère une autonomie par rapport à l'auteur. À la perte d'univocité du récit s'ajoute l'importance accrue du doute vis-à-vis de la Tradition, soit par la confrontation de points de vue divergents, soit par l'adoption d'un ton comique qui vient miner le prestige des auctoritates. Sur ce point, les romans de Rabelais apparaissent comme un champ d'expérimentation intéressant, où la parole, loin d'être disqualifiée, révèle un lien problématique entre le discours et la vérité. Dès lors, plutôt que de conclure hâtivement à un relativisme général chez l'auteur du Tiers livre, faut-il reconsidérer cette « rhétorique de l'aporie », qui, en mettant en équivalence des énoncés contradictoires, conduit le lecteur à une quête du sens plus authentique, parce que non univoque, et promeut une véritable activité de décodage chez le destinataire. L'ultime chapitre de cette étude vient une fois de plus nous donner des outils d'analyse susceptibles d'enrichir notre confrontation personnelle à ces œuvres. Aux considérations théoriques se substitue alors une approche pragmatique rappelant l'importance à accorder au dialogisme comme protocole de lecture, à la dispositio et aux « implications réflexives de l'organisation du récit $\gg(403)$.

Deux utiles annexes viennent compléter cette riche et importante étude : l'une, consacrée aux romans de chevalerie ou d'aventure sur le mode médiéval (447-454), l'autre dévolue aux romans antiques, sentimentaux et pastoraux (455-457). L'abondante bibliographie (461-489) et les index d'auteurs et d'œuvres (491-498) et de notions (499-500) fournissent enfin les instruments nécessaires à l'approfondissement de la notion de genre romanesque à laquelle nous invite cette rigoureuse et féconde contribution. Signalons pour finir quelques coquilles, qui n’altèrent en rien l'ensemble : lire « ni ouverte ni concertée » (213); ajouter la négation initiale «si l'écriture des quatre romanciers n'est pas fidèle au réel » (311); introduire le verbe dans « Il est même possible de relever la formulation $[. ..] \gg(369)$.

THIER RY VICTORIA, CEMAR, Université de Picardie-Jules Verne. 